\title{
Influência de um nutracêutico no sêmen, testosterona, cortisol, eritrograma e peso corpóreo em touros jovens Bos taurus indicus
}

\author{
Influency of one nutraceutic on semen, testosterone, cortisol, \\ eritrogram and body weight in young bulls Bos taurus indicus
}

\author{
Marcelo George Mungai Chacur ${ }^{1 *}$; Paulo Tiago Ferreira Aurélioº; \\ Eunice $\mathrm{Oba}^{3}$; Cecília Braga Laposy ${ }^{4}$; Orivaldo Scalon Júnior ${ }^{5}$; \\ Leandro Inague ${ }^{5}$ Sérgio do Nascimento Kronka ${ }^{6}$
}

\begin{abstract}
Resumo
O presente trabalho teve o objetivo de estudar a influência de um nutracêutico (Promater ${ }^{\circledR}$ ) no sêmen, peso corpóreo, eritrograma e nos hormônios testosterona e cortisol, em touros jovens da raça Nelore. Foram utilizados 20 touros da raça Nelore, com idades entre 30 e 36 meses, divididos em grupo 1 $(\mathrm{G} 1)=10$ (controle) e grupo $2(\mathrm{G} 2)=10$ (grupo nutracêutico (Promater ${ }^{\circledR}$ ): $20 \mathrm{~mL} /$ animal/dia, durante 70 dias). Peso corpóreo, amostras de sangue para análises do eritrograma, testosterona e cortisol; foram obtidos nos dias zero (D0), 35 (D35) e 70 (D70) nos dois grupos. Com relação ao peso, houve diferença $(\mathrm{P}<0,01)$ entre dias $(0,35$ e 70) de colheita com médias de 424,30 kg, 480,70 kg e 502,60 kg, respectivamente, sem diferença significativa entre grupos. Não houve diferença $(\mathrm{P}>0,05)$ entre grupos para o peso corpóreo, eritrograma; quadro espermático, cortisol $(\mathrm{G} 1=3,19 \mu \mathrm{g} / \mathrm{dL}$ e $\mathrm{G} 2=2,91 \mu \mathrm{g} / \mathrm{dL}) \mathrm{e}$ testosterona ( $\mathrm{G} 1=247,48 \mathrm{ng} / \mathrm{dL}$ e G2 $=509,65 \mathrm{ng} / \mathrm{dL})$. Conclui-se que o nutracêutico não influenciou nos níveis séricos de testosterona, cortisol e ganho de peso. Os parâmetros sanguíneos e do sêmen não sofreram alterações devido ao nutracêutico.
\end{abstract}

Palavras-chave: Touro zebu, sêmen, hormônios, sangue, nutrição

\begin{abstract}
The purpose of this study was to investigate the nutraceutic (Promater ${ }^{\circledR}$ ) influence on semen, body weight, eritrogram and hormones testosterone and cortisol in Nelore young bulls. Twenty Nelore bulls were utilized, aging between 30 and 36 months, divided in group 1 (G1)=10 (control) and group 2 $(\mathrm{G} 2)=10$ (nutraceutic group - Promater ${ }^{\circledR}: 20 \mathrm{~mL} /$ animal/day, during 70 days). Corporal weight, blood samples to eritrogram, testosterone and cortisol were obtained in days zero (D0), 35 (D35) and 70 (D70) in two groups. There was difference $(\mathrm{P}<0.01)$ among days collected $(0,35$ and 70$)$ with means of 424.30 $\mathrm{kg}, 480.70 \mathrm{~kg}$ and $502.60 \mathrm{~kg}$, respectively, but there was not difference between groups. There was not difference among groups to semen, eritrogram, cortisol ( $\mathrm{G} 1=3,19 \mu \mathrm{g} / \mathrm{dL} ; \mathrm{G} 2=2,91 \mu \mathrm{g} / \mathrm{dL})$ and testosterone $(\mathrm{G} 1=274,48 \mathrm{ng} / \mathrm{dL} ; \mathrm{G} 2=509,65 \mathrm{ng} / \mathrm{dL})$. The results exposed allow concluding that were not observed significative effect of the nutraceutic upon evaluated parameters: body weight, semen, hormonal profile and eritrogram.
\end{abstract}

Key words: Zebu bull, semen, hormones, blood, nutrition.

\footnotetext{
1 Médico Veterinário - Prof. Dr. Universidade do Oeste Paulista - UNOESTE, Rod. Raposo Tavares, km 572, Campus II, CEP: 19.067-175, Presidente Prudente, SP - Tel: (18) 3229-2077, FAX: (18) 3229-2080, E-mail: chacur@unoeste.br

2 Médico Veterinário - Mestre em Ciência Animal pela UNOESTE. E-mail: ptaurelio@hotmail.com

3 Médica Veterinária - Profa. Dra. FMVZ-UNESP-Botucatu, SP. E-mail: euniceoba@fmvz.unesp.br

4 Médica Veterinária - Profa. Dra. FCA-UNOESTE. E-mail: claposy@unoeste.br

5 Ex-alunos de Iniciação Científica - UNOESTE. E-mail: oscalo@hotmail.com ; inague@hotmail.com

6 Prof. Dr. - FCAVJ-UNESP, Jaboticabal, SP. E-mail: kronka@hotmail.com

* Autor para correspondência
} 


\section{Introdução}

A bovinocultura de corte sofre influência da nutrição, afetando o desempenho dos touros. A despeito do mercado ofertar uma gama de suplementos nutricionais para a espécie bovina, a opinião pública compartilha da idéia da utilização de produtos fornecidos aos animais, cujas formulações não contenham ingredientes que necessitem de período de carência para o consumo humano da carne. Dentro desse raciocínio, os nutracêuticos figuram como uma opção para a suplementação nutricional nos bovinos.

Os nutracêuticos geralmente são constituídos por vitaminas, minerais e ácidos graxos, sendo a vitamina A um nutriente essencial e sua necessidade na reprodução dos animais está bem estabelecida, atuando na função celular imune (NATIONAL ACADEMY OF SCIENCES, 2002), em camundongos, a vitamina A colabora com a produção de hormônios esteróides (WELLIK; DELUCA, 1996) atua também na formação dos espermatozóides nos bovinos (RODE et al., 1995). As vitaminas do complexo B servem com co-fatores na maioria das vias metabólicas, sendo necessárias para o crescimento corpóreo, reprodução e desenvolvimento do feto (HURLEY; DOANE, 1989).

No Brasil foram detectados níveis baixos de selênio (Se) em algumas regiões do estado de São Paulo (LUCCI et al., 1984). O selênio e a vitamina E agem como antioxidantes nas células, sendo o Se parte da enzima glutationa peroxidase, presente no citoplasma e na matriz mitocondrial, reduzindo o dano oxidativo causado pelos radicais livres em proteínas e ácidos nucléicos; a glutationaperoxidase contém selênio e forma a segunda linha de defesa de proteção das membranas celulares depois da vitamina E (MacDONALD; EDWARDS; GREENHALGH, 2002). Há uma complementação no sítio de ação, onde o selênio atua no meio intracelular e a vitamina $\mathrm{E}$ no meio extracelular (PASCHOAL; ZANETTI; CUNHA, 2003)
$\mathrm{O}$ microelemento zinco funciona como um ativador e constituinte de mais de 30 metaloenzimas que estão envolvidas no metabolismo dos ácidos nucléicos e carboidratos, além da síntese de proteínas (NRC, 2001). O zinco participa na produção, armazenagem e secreção de hormônios, bem como ativador de receptores para a resposta dos órgãos. Os principais efeitos do zinco são a produção e secreção de testosterona, insulina e corticóides da adrenal (McDOWELL; EDWARDS; GREENHALGH, 1992).

A testosterona é essencial à função reprodutiva nos machos, atua estimulando os estádios finais da espermatogênese; prolonga a vida útil dos espermatozóides no epidídimo e estimula o crescimento, o desenvolvimento e a atividade secretora dos órgãos sexuais do macho, exteriorizando as características sexuais secundárias, segundo Hafez (2004), sendo necessária para a manutenção da capacidade de serviço (BLOCKEY; GALLOWAY, 1978).

As hipóteses formuladas foram: 1 - de que o nutracêutico influenciasse na elevação do nível sérico de testosterona, aumentando o peso corpóreo dos touros jovens; 2 - de que o nutracêutico influenciasse na elevação do nível sérico de cortisol.

O presente trabalho teve o objetivo de estudar a influência de um nutracêutico (Promater ${ }^{\circledR}$ ) no sêmen, peso corpóreo, eritrograma e nos hormônios testosterona e cortisol, em touros jovens da raça Nelore.

\section{Material e Métodos}

Foram utilizados 20 touros jovens e dóceis da raça Nelore, com idades entre 30 e 36 meses, com escore de condição corporal entre 3,0 e 3,5 (1 = muito magro a 5 = muito gordo), segundo escala proposta por Lowman, Scott e Somervalle (1976). Os pesos médios iniciais eram de $418 \pm 6 \mathrm{~kg}$ para o grupo 1 e de $430 \pm 5 \mathrm{~kg}$ para o grupo 2 . Os touros foram divididos em dois grupos: grupo $1(\mathrm{G} 1$ controle $)=10$ animais 
que receberam $20 \mathrm{~mL} /$ animal/dia de solução salina via oral como placebo e grupo 2 (G2 suplementado) $=10$ animais, suplementados com nutracêutico ${ }^{7}$ (Promater ${ }^{\circledR}$ ) na dose de $20 \mathrm{~mL} / \mathrm{animal} / \mathrm{dia}$, via oral, durante 70 dias, entre os meses de agosto e outubro, criados em propriedade pertencente ao município de Presidente Prudente - SP. Cada grupo foi alojado em seu respectivo piquete com áreas iguais de 3ha formados com Brachiaria decumbens e arborizados, fornecendo sombra aos touros de ambos os grupos. Os dois grupos receberam ração total depositada em seus respectivos cochos com $10 \mathrm{~m}$ lineares, alimentação essa constituída por cana-de-açúcar triturada (70\%) 21kg/animal/dia, polpa cítrica $(30 \%) 9 \mathrm{~kg} / \mathrm{animal} / \mathrm{dia}$, sal proteinado (200g/animal/dia), sal mineral em separado e água "ad libitum". A ração total foi fracionada em duas partes iguais, fornecida às $8: 00 \mathrm{~h}$ e 17:00h, de forma que o seu total fosse consumido, sem que os animais espalhassem o alimento.

A formulação do nutracêutico (Promater ${ }^{\circledR}$ ) utilizado, segundo informações do fabricante é composta por: vitamina A - 804.170 UI, vitamina B12 - $16.650 \mu \mathrm{g}$, vitamina E - 43.425 UI, ácido linolênico - $46.518 \mathrm{mg}$, ácido linoléico - 267.278 $\mathrm{mg}$, ácido oléico - $113.103 \mathrm{mg}$, ácido fólico $20.000 \mathrm{mg}$, L-carnitina - $100.780 \mathrm{mg}$, L-lisina $10.078 \mathrm{mg}$, DL metionina - $10.078 \mathrm{mg}, \beta$-caroteno - $5.020 \mathrm{mg}$, zinco $4.580 \mathrm{mg}$, selênio $152 \mathrm{mg}$ e veículo q.s.p. $1000 \mathrm{~g}$. O produto vem pronto para uso, apresentando aspecto físico viscoso.

Foram colhidas três amostras de sêmen e sangue para a padronização dos dados, antes das coletas efetivas para o presente estudo. Posteriormente, amostras de sêmen, sangue para o eritrograma, testosterona e cortisol foram coletadas, bem como a pesagem dos animais foram obtidas nos dias zero (D0), 35 (D35) e 70 (D70) nos dois grupos. As amostras de sêmen foram coletadas com eletroejaculador automático ${ }^{8}$ e analisadas segundo

\footnotetext{
7 Nutracêutico - Promater $^{\circledR}$ - Vetnil

8 Eletroejaculador automático - AUTOEJAC - NEOVET, Uberaba-MG.
}

normas do CBRA (1998). O sangue foi colhido por venopunção jugular com o sistema (Vacutainer®) entre 7:00h e 8:00h. Após centrifugação $(1.500 \mathrm{~g}$ por 15 minutos), o plasma para as dosagens de testosterona e cortisol foi armazenado em criotubos (Eppendorf®), congelados a $-20^{\circ} \mathrm{C}$ até o processamento com "kits" comerciais DPC-Medlab, em fase sólida, por radioimunoensaio (RIA), em duplicata, no Laboratório de Endocrinologia do Departamento de Reprodução Animal e Radiologia Veterinária da FMVZ, UNESP, Botucatu - SP.

O eritrograma consistiu da contagem total de hemácias ${ }^{1}$, concentração da hemoglobina ${ }^{9}$; e determinação do volume globular ${ }^{10}$. Foram calculados os índices hematimétricos do volume corpuscular médio (VCM) e concentração da hemoglobina corpuscular média (CHCM).

Os dados experimentais foram analisados em esquema fatorial inteiramente casualizado, com os fatores: grupos (1 e 2) e colheitas (1, 2 e 3), com números iguais de repetições, para as variáveis consideradas. Utilizou-se o delineamento inteiramente casualizado com 60 repetições. Os dados foram submetidos à análise de variância pelo teste $\mathrm{F}$ e as médias comparadas pelo teste de Tukey (BANZATTO; KRONKA, 2006).

\section{Resultados e Discussão}

A despeito do emprego de novas técnicas de suplementação alimentar para animais, os nutracêuticos ainda são utilizados em pequena parcela dos criatórios de bovinos, sendo seus efeitos alvo de pesquisas. Na esfera masculina, existe a necessidade de mais informações sobre a influência dos nutracêuticos na reprodução de touros. O reprodutor bovino não pode ser obeso para que desempenhe sua função de forma adequada, logo, sua alimentação deve ser balanceada mantendo o escore corporal.

\footnotetext{
9 Contador de céluas CELM HB 530 - CELM, São Paulo-SP.

${ }^{10}$ Microcentrífuga FANEN - FANEN, São Paulo-SP.
} 
Houve acréscimo do peso corpóreo médio diário de $1,12 \mathrm{~kg}$ para os grupos, perfazendo ganho total de $15,58 \%$ no peso. Para o peso corpóreo, houve diferença significativa $(\mathrm{P}<0,01)$ entre os dias das colheitas, D70: 502,60 kg em relação aos dias D0: 424,30 kg e D35: 480,70 kg (Tabela 1). Os ganhos de peso dos animais nos dois grupos demonstram que os mesmos tiveram acesso à alimentação, com quantidade e qualidade satisfatórias, levando-se em consideração que o experimento foi conduzido, em sua maior parte, na estação do inverno e primavera. Não houve diferença significativa para o peso entre os grupos, demonstrando que os constituintes do nutracêutico não colaboram para o ganho de peso.

Foi observado desenvolvimento corporal satisfatório dos touros, no presente experimento, similar às observações de Evans (1996), sugerindo que a maturidade sexual está mais intimamente relacionada ao peso do animal do que a idade, sofrendo a influência de fatores como raça, heterose, balanceamento hormonal e manejo. A nutrição influencia a produção hormonal e altera as características de crescimento e reprodução, visto que o consumo de dietas pobres em energia pode reduzir os níveis de testosterona circulante (CHASE et al., 1993).

As percentagens de ganho de peso obtidas nos grupos do presente estudo, foram inferiores às relatadas por Alves et al. (2000) e Jorge, Rosa e Silva (2006), com $22 \%$ e $25 \%$, respectivamente, para bovinos confinados; e Rasteiro, Bremer-Neto e Arenas (2006) com incremento de 19,4\% no peso dos bovinos em pastejo extensivo no período de seca e Arenas et al. (2007) que verificaram 33,3\% de acréscimo no peso. O acréscimo no ganho de peso citado por esses autores foi superior, uma vez que as formulações das rações tinham o objetivo de engordar os animais.

Tabela 1. Médias e desvios-padrão nos grupos G1 controle e G2 suplementado com nutracêutico para o peso corpóreo de touros da raça Nelore, com idades de 30 a 36 meses, Presidente Prudente - SP.

\begin{tabular}{|c|c|}
\hline Fatores & peso corpóreo $(\mathrm{kg})$ \\
\hline \multicolumn{2}{|l|}{ Grupos } \\
\hline G1 controle & $466,26 \pm 5,99^{\text {a }}$ \\
\hline G2 suplementado & $472,13 \pm 4,87^{\text {a }}$ \\
\hline \multicolumn{2}{|l|}{ Dias (D) } \\
\hline D0 & $424,30 \pm 5,45^{\mathrm{c}}$ \\
\hline D35 & $480,70 \pm 6,02^{b}$ \\
\hline D70 & $502,60 \pm 4,73^{\mathrm{a}}$ \\
\hline \multicolumn{2}{|l|}{ Teste F } \\
\hline Grupos (G) & $0,78^{\text {ns }}$ \\
\hline Dias (D) & $0,000 * *$ \\
\hline Interação (GxD) & $0,55^{\mathrm{ns}}$ \\
\hline
\end{tabular}

ns- não significativo ( $\mathrm{P}>0,05)$; *significativo no nível de 5\% $(\mathrm{P}<0,05)$; **significativo no nível de $1 \%(\mathrm{P}<0,01) ; \mathrm{a}, \mathrm{b}-\mathrm{em}$ cada coluna, para cada fator, médias seguidas de mesma letra não diferem $(\mathrm{P}>0,05)$.

Com relação ao espermiograma, não houve diferença significativa entre grupos para volume, motilidade, vigor, turbilhão e defeitos espermáticos maiores, menores e totais (Tabela 2), diferindo dos resultados relatados por Kumar et al. (2006) em tourinhos mestiços com 24 meses de idade e pesos médios de $316 \mathrm{~kg}$, também suplementados com zinco durante seis meses, onde diferenças para volume do ejaculado, motilidade e níveis de testosterona foram obtidas. Novas formulações com zinco devem ser testadas em reprodutores, envolvendo diferentes doses e períodos de fornecimento, visando-se uma padronização. 
$\mathrm{Na}$ espécie suína, machos aos 12 meses de idade apresentaram maior volume e motilidade espermática, quando suplementados previamente durante quatro meses com vitamina do complexo B (AUDET et al., 2004), presente na formulação do nutracêutico do presente estudo. Por outro lado, não houve diferença $(\mathrm{P}>0,05)$ para a morfologia espermática entre grupos, similar aos relatos de Audet et al. (2004).

Provavelmente, o metabolismo espermático dos zebuínos do presente estudo, mostrou-se mais eficiente em relação aos taurinos da raça Limousin (CHACUR; MACHADO NETO; CRISTANCHO, 2006b) e em Canchim, para o mesmo período do ano com idades similares (CHACUR; ARAÚJO; KRONKA, 2006a), taurinos esses cujos ejaculados revelaram valores inferiores para a motilidade e vigor espermáticos, e percentagens maiores para os defeitos dos espermatozóides.

Com relação ao espermiograma, todas as amostras de sêmen dos dois grupos experimentais, revelaram as seguintes características macroscópicas: coloração branca marmórea e aspecto viscoso, não sendo verificada qualquer variação entre as colheitas e grupos. Houve diferença significativa $(\mathrm{P}<0,05)$ entre dias de coleta para os defeitos menores e totais. $\mathrm{O}$ vigor espermático foi inferior à média de 4,3 relatada por Chacur, Sanches-Martinez e Machado Neto (2006c) em Nelores com cinco a seis anos de idade na primavera. Essa superioridade pode estar relacionada à maior faixa etária, propiciando produção espermática plena.

O volume dos ejaculados foi superior ao descrito por Silva et al. (2002) de 4,0 $\mathrm{mL}$ e inferior aos 12,0 mL citado por Martinez et al. (2000), estando esse aspecto quantitativo sujeito a variação, principalmente frente ao método de coleta por meio da eletroejaculação, onde os estímulos nas glândulas sexuais acessórias são de freqüência e intensidade variáveis .

As médias para a motilidade espermática foram inferiores às relatadas por Silva (1993) com 65,3\%;
Sarreiro (2002) obtendo 62,7\% em touros zebuínos e Chacur, Sanches-Martinez e Machado Neto (2006c) com média de $75 \%$ em Nelores na pré-estação de monta. Para os defeitos maiores, as médias foram superiores ao limite de $10 \%$, preconizado pelo Colégio Brasileiro de Reprodução Animal (CBRA, 1998), estando os defeitos menores e totais dentro dos padrões recomendados. Sugere-se que a inatividade sexual dos touros possa ter influenciado na percentagem de defeitos maiores (Tabela 2).

A morfologia espermática supostamente é influenciada pelos constituintes protéicos do plasma seminal, os quais variam conforme a estação do ano e componentes da alimentação fornecida, repercutindo na fertilidade de touros Limousin, criados em regime semi-intensivo (RABESQUINE; CHACUR; GARCIA, 2003; CHACUR; RABESQUINE; MACHADO NETO, 2003; 2004; CHACUR; MACHADO NETO, 2007); na raça Canchim aos 14 e 48 meses de idade (CHACUR; ARAÚJO; KRONKA, 2006a) e na raça Nelore, criada extensivamente (SANCHEZ et al., 2004; CHACUR; SANCHEZ-MARTINEZ; MACHADO NETO, 2006c).

Comparando-se os grupos 1 e 2, controle e suplementado, respectivamente, observou-se que houve diferença significativa para glóbulos vermelhos $(\mathrm{GV})(\mathrm{p}<0,05)$ entre os dias 0 e 70 do experimento, com redução de $7,8 \times 10^{6} / \mathrm{mm}^{3}$ no dia zero para $7,0 \times 10^{6} / \mathrm{mm}^{3}$, permanecendo dentro dos valores normais para bovinos. Com relação aos dias de coleta, verificou-se diferenças significativas para a variável hemoglobina $(\mathrm{Hb})$, volume globular (VG), volume corpuscular médio (VCM) e concentração de hemoglobina corpuscular média (CHCM) (Tabela 3). No presente estudo, valores inferiores para $\mathrm{Hb}, \mathrm{VG}$ e $\mathrm{VCM}$ foram obtidos em relação aos índices de $\mathrm{Hb}=12,8 \mathrm{~g} / \mathrm{dL}, \mathrm{VG}=41,2 \%$ e $\mathrm{VCM}=61,83 \mathrm{fL}$, relatado para novilhas da raça Aquitânica (5/8 Blond"Aquitaine x 3/8 Caracu) acima de 12 meses de idade, criadas em regime semi-intensivo, suplementadas com milho, soja e algodão (DIAS JÚNIOR et al., 2006). Esses 
valores inferiores, supostamente, estão associados à conversão alimentar, sendo essa superior nas raças de origem européia e nos seus mestiços. Por outro lado, nos touros Nelore o valor para o CHCM foi similar aos $31,41 \%$ observados nas fêmea da raça
Aquitânica (DIAS JÚNIOR et al., 2006). Para as hemácias $(\mathrm{GV})$, nos Nelores, valor superior foi observado em relação às fêmeas $\mathrm{GV}=6,68 \times 10^{6} \%$ mm³ (DIAS JÚNIOR et al., 2006).

Tabela 2. Médias e desvios-padrão nos grupos G1 controle e G2 suplementado com nutracêutico para as seguintes características: volume (Vol), motilidade (Mot), vigor (Vig), turbilhonamento (Turb), defeitos maiores (DM), defeitos menores (Dm) e defeitos totais (DT); de touros da raça Nelore, com idades de 30 a 36 meses, Presidente Prudente - SP.

\begin{tabular}{|c|c|c|c|c|c|c|c|}
\hline Fatores & Vol (mL) & Mot (\%) & $\begin{array}{c}\text { Vig } \\
(1 \text { a } 5)\end{array}$ & $\begin{array}{l}\text { Turb } \\
(1 \text { a } 5)\end{array}$ & DM (\%) & $\operatorname{Dm}(\%)$ & DT (\%) \\
\hline \multicolumn{8}{|l|}{ Grupos } \\
\hline G1 controle & $6,13 \pm 2,32^{\mathrm{a}}$ & $54,99 \pm 7,44^{\text {a }}$ & $1,95 \pm 0,18^{\mathrm{a}}$ & $1,93 \pm 0,17^{\text {a }}$ & $15,84 \pm 6,02^{\text {a }}$ & $19,98 \pm 5,99$ a & $26,92 \pm 7,02$ a \\
\hline G2suplementado & $7,03 \pm 2,73^{\mathrm{a}}$ & $55,38 \pm 7,23^{\text {a }}$ & $1,96 \pm 0,16^{\mathrm{a}}$ & $1,96 \pm 0,18^{a}$ & $17,5 \pm 6,35^{\text {a }}$ & $20,55 \pm 5,87^{\mathrm{a}}$ & $27,71 \pm 7,35^{\mathrm{a}}$ \\
\hline \multicolumn{8}{|l|}{ Dias (D) } \\
\hline D0 & $5,91 \pm 2,21^{\mathrm{a}}$ & $54,86 \pm 7,12^{\mathrm{a}}$ & $1,97 \pm 0,19^{\text {a }}$ & $1,99 \pm 0,19^{\text {a }}$ & $16,75 \pm 6,21^{\text {a }}$ & $18,16 \pm 5,53^{\mathrm{b}}$ & $25,48 \pm 7,24^{b}$ \\
\hline D35 & $7,66 \pm 3,01^{\mathrm{a}}$ & $56,45 \pm 7,05^{\mathrm{a}}$ & $1,96 \pm 0,18^{\text {a }}$ & $1,85 \pm 0,18^{\text {a }}$ & $15,96 \pm 6,02^{\mathrm{a}}$ & $19,51 \pm 5,63^{\mathrm{ab}}$ & $26,26 \pm 7,44^{a b}$ \\
\hline D70 & $6,17 \pm 2,18^{\text {a }}$ & $54,25 \pm 6,99^{\text {a }}$ & $1,95 \pm 0,18^{\text {a }}$ & $1,98 \pm 0,17^{\text {a }}$ & $17,44 \pm 6,40^{\text {a }}$ & $23,14 \pm 6,02^{\text {a }}$ & $30,20 \pm 7,95^{\text {a }}$ \\
\hline \multicolumn{8}{|l|}{ Teste $\mathrm{F}$} \\
\hline Grupos (G) & $0,38^{\mathrm{ns}}$ & $0,86^{\mathrm{ns}}$ & $0,80^{\mathrm{ns}}$ & $0,66^{\mathrm{ns}}$ & 0,29 ns & 0,77 ns & 0,73 ns \\
\hline Dias (D) & $0,06^{\mathrm{ns}}$ & $0,69^{\text {ns }}$ & $0,94^{\mathrm{ns}}$ & $0,27^{\mathrm{ns}}$ & 0,63 ns & $0,01 *$ & $0,025^{*}$ \\
\hline Interação (GxD) & $0,37^{\mathrm{ns}}$ & 0,83 ns & $0,31 \mathrm{~ns}$ & $0,52 \mathrm{~ns}$ & $0,20^{\mathrm{ns}}$ & $0,74 \mathrm{~ns}$ & $0,87^{\mathrm{ns}}$ \\
\hline
\end{tabular}

ns- não significativo $(\mathrm{P}>0,05)$; *significativo no nível de $5 \%(\mathrm{P}<0,05)$; **significativo no nível de $1 \%(\mathrm{P}<0,01)$. $\mathrm{a}, \mathrm{b}$ - em cada coluna, para cada fator, médias seguidas de mesma letra não diferem $(\mathrm{P}>0,05)$.

Tabela 3. Médias e desvios-padrão para glóbulos vermelhos (GV), hemoglobina (Hb), volume globular (VG), volume corpuscular médio (VCM) e concentração de hemoglobina corpuscular média (CHCM) de touros da raça Nelore, com idades entre 30 e 36 meses, Presidente Prudente-SP.

\begin{tabular}{lccccc}
\hline \multicolumn{1}{c}{ Eritrograma } & $\begin{array}{c}\mathrm{GV} \\
\left(10^{6} / \mathrm{mm}^{3}\right)\end{array}$ & $\begin{array}{c}\mathrm{Hb} \\
(\mathrm{g} / \mathrm{dL})\end{array}$ & $\begin{array}{c}\mathrm{VG} \\
(\%)\end{array}$ & $\begin{array}{c}\text { VCM } \\
(\mathrm{fL})\end{array}$ & $\begin{array}{c}\text { CHCM } \\
(\%)\end{array}$ \\
\hline Grupo 1(controle) & $7,5 \pm 1,2^{\mathrm{A}}$ & $12,0 \pm 1,9^{\mathrm{A}}$ & $36,1 \pm 2,9^{\mathrm{A}}$ & $48,1 \pm 3,5^{\mathrm{A}}$ & $33,2 \pm 1,9^{\mathrm{A}}$ \\
Grupo 2 (suplementado) & $7,5 \pm 1,4^{\mathrm{A}}$ & $11,8 \pm 1,7^{\mathrm{A}}$ & $35,6 \pm 1,9^{\mathrm{A}}$ & $47,6 \pm 3,2^{\mathrm{A}}$ & $33,1 \pm 1,6^{\mathrm{A}}$ \\
Dia 0 & $7,8 \pm 1,5^{\mathrm{A}}$ & $12,1 \pm 1,5^{\mathrm{A}}$ & $36,6 \pm 2,4^{\mathrm{A}}$ & $51,9 \pm 3,7^{\mathrm{A}}$ & $34,5 \pm 1,5^{\mathrm{A}}$ \\
Dia 35 & $7,5 \pm 1,1^{\mathrm{AB}}$ & $12,0 \pm 1,3^{\mathrm{A}}$ & $35,6 \pm 2,7^{\mathrm{AB}}$ & $47,1 \pm 3,1^{\mathrm{B}}$ & $33,9 \pm 1,7^{\mathrm{A}}$ \\
Dia70 & $7,0 \pm 0,9^{\mathrm{B}}$ & $11,4 \pm 1,6^{\mathrm{B}}$ & $35,4 \pm 2,5^{\mathrm{B}}$ & $44,8 \pm 3,9^{\mathrm{B}}$ & $31,3 \pm 1,4^{\mathrm{B}}$ \\
Valores normais & $5,0-10,0$ & $8,0-14,0$ & $24,0-48,0$ & $40,0-60,0$ & $30,0-36,0$ \\
\hline Teste F & & & & & \\
Grupos (G) & $7,21^{* *}$ & $28 \mathrm{~ns}$ & $0.20 \mathrm{~ns}$ & $0.31 \mathrm{~ns}$ & $0.04 \mathrm{~ns}$ \\
Dias (D) & $00 \mathrm{~ns}$ & $7.32^{* *}$ & $3.82^{*}$ & $20.04^{* *}$ & $44.11^{* *}$ \\
Interação (GxD) & $08 \mathrm{~ns}$ & $11 \mathrm{~ns}$ & $0.03 \mathrm{~ns}$ & $0.12 \mathrm{~ns}$ & $0.33 \mathrm{~ns}$
\end{tabular}

Letras iguais indicam que não houve diferenças significativas entre grupos $(\mathrm{p}>0,05)$ pelo teste $\mathrm{F}$. ns $=$ não significativo. ${ }^{*}=$ significativo ao nível de $5 \%(\mathrm{p}<0,05)$. ${ }^{* *}=$ significativo ao nível de $1 \%(\mathrm{p}<0,01)$. 
Ao interagir grupos e dias, não foi verificada elevação numérica para os parâmetros sanguíneos ao longo dos dias do experimento (Tabela 3), dessa forma o nutracêutico testado não influenciou de forma significativa na produção de novas células sanguíneas, apesar de conter em sua formulação $4.580 \mathrm{mg} / \mathrm{kg}$ de zinco, $20.000 \mathrm{mg} / \mathrm{kg}$ de ácido fólico e $16.650 \mu \mathrm{g}$ de vitamina B12 que colaboram para a hematopoiese. $\mathrm{O}$ zinco protege as membranas biológicas dos efeitos antioxidantes, estando presente nos eritrócitos (FELDMAN; ZINKL; JAIN, 2000). Nessa mesma linha de pesquisa, Wang et al. (2006) estudaram a influência de altos níveis de zinco e vitamina B12 em cordeiros, revelando elevação dos parâmetros sanguíneos.
Para os níveis séricos de cortisol, não houve diferença significativa entre os grupos com valores de $3,19 \mu \mathrm{g} / \mathrm{dL}$ no Grupo 1 e 2,91 $\mu \mathrm{g} / \mathrm{dL}$ no Grupo $2(\mathrm{CV}=47,34 \%)$. Entre os dias de colheita de sangue, houve diferença significativa $(\mathrm{P}<0,01)$ para o cortisol (Tabela 4), podendo estar relacionado com a adaptação dos touros ao ambiente e ao manejo nutricional recebido. Na segunda metade do experimento verificou-se que os animais permitiam a aproximação das pessoas, mesmo durante a ingestão do alimento fornecido no cocho. Com relação aos níveis séricos de testosterona para o Grupo 1 e Grupo 2 de 274,48 ng/dL e 509,69 ng/dL, $(\mathrm{CV}=148.40 \%)$, respectivamente (Tabela 4$)$.

Tabela 4. Médias e desvios-padrão para cortisol e testosterona em de touros da raça Nelore, com idades de 30 a 36 meses, Presidente Prudente - SP.

\begin{tabular}{lcc}
\hline Fatores & Cortisol & Testosterona \\
\hline Grupos & $3,19 \pm 0,2^{\mathrm{a}}$ & $274,48 \pm 13.45^{\mathrm{a}}$ \\
G1 (controle) & $2,91 \pm 0,1^{\mathrm{a}}$ & $509,65 \pm 19,64^{\mathrm{a}}$ \\
G2 (suplementado) & & \\
\hline Dias (D) & $3.81 \pm 0,4^{\mathrm{a}}$ & $181.71 \pm 7,45^{\mathrm{b}}$ \\
D0 & $3.06 \pm 0,3^{\mathrm{b}}$ & $441.42 \pm 12,01^{\mathrm{a}}$ \\
D35 & $2.28 \pm 0,2^{\mathrm{c}}$ & $553.13 \pm 13,21^{\mathrm{a}}$ \\
D70 & & $2.45^{\mathrm{ns}}$ \\
\hline Teste F & $57^{\mathrm{ns}}$ & $9.55^{* *}$ \\
Grupos (G) & $17.64^{* *}$ & $2.05^{\mathrm{ns}}$ \\
Dias (D) & $98^{\mathrm{ns}}$ & \\
Interação (GxD) &
\end{tabular}

ns- não significativo; *significativo no nível de $5 \%(\mathrm{P}<0,05) ; * *$ significativo no nível de $1 \%(\mathrm{P}<0,01)$.

A produção de testosterona pode ser um indicador da fertilidade em touros jovens, por estar relacionada com a idade à puberdade (POST; CHRISTENSEN; SEIFERT, 1987). Os níveis séricos de testosterona do Grupo 1 foram inferiores aos descritos em Nelore de 293,72 $\pm 18,25 \mathrm{ng} / \mathrm{dL}$ (LEZIER, 2004), por outro lado, foi superior às médias de $159 \mathrm{ng} / \mathrm{dL}$ para a raça Nelore (SANTOS, 1999); 104,4 ng/dL nas raças Santa Gertrudis e Nelore (SANCHES, 1999) e em touros da raça Nelore, criados extensivamente, com idades entre 24 e 36 meses, com 250 ng/dL (OBA, 1985).

Não houve diferença significativa para a testosterona entre os grupos 1 e 2, mas observa-se que o nível sérico de andrógeno foi numericamente superior no grupo 2, tratado com nutracêutico, com média de 509,65 ng/dL, perante 274,48 ng/dL no grupo 1 que não recebeu o produto. $\mathrm{O}$ resultado obtido no grupo suplementado com nutracêutico 
foi superior aos valores médios de $317 \mathrm{ng} / \mathrm{dL}$ (SANCHES et al., 1998) e $400 \mathrm{ng} / \mathrm{dL}$ em zebus aos 39 meses de idade (BARBOSA et al., 1992). A elevação do nível sérico de testosterona no grupo suplementado com nutracêutico pode estar relacionada com o microelemento zinco, descrito por McDowell, Edwards e Greenhalgh (1992) como ativador na produção e secreção de testosterona. Possivelmente, se o experimento fosse mantido por um período mais longo, o nível sérico de testosterona no grupo tratado com nutracêutico continuaria a se elevar, resultando em diferença significativa.

A secreção de andrógenos nos animais, aparentemente não é afetada pela exposição dos testículos ao calor (SETCHELL, 2006), o mesmo foi observado para búfalos nascidos e criados extensivamente, quando mantidos em câmara bioclimática a $39^{\circ} \mathrm{C}$, durante 54 dias (CHACUR, 2000; CHACUR; OBA, 2005). Supostamente, ocorre uma adaptação do organismo dos animais nascidos e criados em regiões quentes. Houve queda na testosterona de touros Nelores submetidos à insulação escrotal, com aumento do nível hormonal após um período de adaptação (GABALDI, 2000).

Os níveis de testosterona encontrados na literatura consultada variam dentro da mesma raça e da faixa etária dos machos bovinos, podendo estar relacionado às diferentes composições nutricionais, precocidade das linhagens, momento das colheitas de sangue e do ganho de peso corpóreo.

Verificou-se correlação entre cortisol e eritrograma no dia 35 do G1 (controle) para hemácias e hemoglobina $(\mathrm{Hb})$; e entre cortisol e volume globular (VG) no dia zero. Para o grupo de animais suplementados com nutracêutico reprodutivo, as correlações entre testosterona e hemograma; e testosterona e cortisol não foram significativas (Tabelas 5 e 6), dessa forma sugere-se a não influência do nutracêutico nessas características.

No presente estudo, houve correlação entre testosterona e eritrograma no dia zero do G1 (controle) para hemácias e hemoglobina $(\mathrm{Hb})$, e entre testosterona e volume globular (VG) no dia 0 . Para o dia 35 do G1, houve correlação entre testosterona e hemácias e entre testosterona e volume corpuscular médio (VCM) (Tabela 6).

Tabela 5. Coeficientes de correlação ( $r$ ) entre o cortisol e o eritrograma de touros (G1 controle) e tratados com nutracêutico (G2 suplementado) da raça Nelore com idades entre 30 e 36 meses, Presidente Prudente, SP.

\begin{tabular}{|c|c|c|c|c|c|}
\hline Grupos & Hemácias & $\mathrm{Hb}$ & $\mathrm{VG}$ & VCM & $\mathrm{CHCM}$ \\
\hline \multicolumn{6}{|l|}{ G1 } \\
\hline Dia 0 & $0,24^{\mathrm{ns}}$ & $0,21^{\mathrm{ns}}$ & $0,22^{\mathrm{ns}}$ & $-0,13^{\mathrm{ns}}$ & $-0,04^{\mathrm{ns}}$ \\
\hline Dia 35 & $0,70^{*}$ & $0,72^{*}$ & $0,68^{*}$ & $-0,61^{\mathrm{ns}}$ & $0,34^{\mathrm{ns}}$ \\
\hline Dia 70 & $0,46^{\mathrm{ns}}$ & $0,56^{\mathrm{ns}}$ & $0,61^{\text {ns }}$ & $-0,12^{\mathrm{ns}}$ & $0,09^{\text {ns }}$ \\
\hline \multicolumn{6}{|l|}{ G2 } \\
\hline Dia 0 & $0,18^{\mathrm{ns}}$ & $0,19^{\text {ns }}$ & $0,33^{\text {ns }}$ & $0,15^{\mathrm{ns}}$ & $-0,21^{\mathrm{ns}}$ \\
\hline Dia 35 & $-0,07^{\mathrm{ns}}$ & $-0,08^{\mathrm{ns}}$ & $0,07^{\mathrm{ns}}$ & $0,17^{\text {ns }}$ & $-0,36^{\mathrm{ns}}$ \\
\hline Dia 70 & $-0,14^{\mathrm{ns}}$ & $0,03^{\mathrm{ns}}$ & $0,07^{\mathrm{ns}}$ & $0,28^{\mathrm{ns}}$ & $-0,09^{\mathrm{ns}}$ \\
\hline
\end{tabular}

ns- não significativo; *significativo no nível de $5 \%(\mathrm{P}<0,05)$. 
Tabela 6. Coeficientes de correlação (r) de testosterona com hemograma de touros controle (grupo1) e tratados com nutracêutico (grupo2) da raça Nelore com idades entre 30 e 36 meses, Presidente Prudente, SP.

\begin{tabular}{|c|c|c|c|c|c|}
\hline Grupos & Hemác. & $\mathrm{Hb}$ & VG & VCM & CHCM \\
\hline \multicolumn{6}{|l|}{ G1 } \\
\hline Dia 0 & $-0,64^{*}$ & $-0,70^{*}$ & $-0,81^{* *}$ & $-0,04^{\mathrm{ns}}$ & $-0,34^{\text {ns }}$ \\
\hline Dia 35 & $-0,81^{* *}$ & $-0,51^{\mathrm{ns}}$ & $-0,56^{\mathrm{ns}}$ & $0,93^{* *}$ & $-0,19^{\text {ns }}$ \\
\hline Dia 70 & $0,09^{\mathrm{ns}}$ & $-0,12^{\mathrm{ns}}$ & $-0,59^{\mathrm{ns}}$ & $-0,35^{\mathrm{ns}}$ & $0,35^{\mathrm{ns}}$ \\
\hline \multicolumn{6}{|l|}{ G2. } \\
\hline Dia 0 & $-0,11^{\text {ns }}$ & $0,09^{\mathrm{ns}}$ & $-0,10^{\mathrm{ns}}$ & $0,07^{\mathrm{ns}}$ & $0,39^{\mathrm{ns}}$ \\
\hline Dia 35 & $-0,23^{\mathrm{ns}}$ & $-0,26^{\mathrm{ns}}$ & $-0,08^{\mathrm{ns}}$ & $0,43^{\mathrm{ns}}$ & $-0,42^{\mathrm{ns}}$ \\
\hline Dia 70 & $-0,28^{\text {ns }}$ & $-0,43^{\text {ns }}$ & $-0,43^{\text {ns }}$ & $0,12^{\mathrm{ns}}$ & $-0,16^{\mathrm{ns}}$ \\
\hline
\end{tabular}

ns- não significativo; *significativo no nível de 5\% (P<0,05); ** significativo no nível de $1 \%(\mathrm{P}<0,01)$.

A utilização dos nutracêuticos em bovinos é uma opção para suplementá-los, devendo ser objetivo de um maior número de estudos para a padronização de protocolos mutricionais para reprodutores. Sugerese que novas formulações sejam testadas visando a padronização, com períodos de fornecimento mais longos para uma maior compilação de informações.

\section{Conclusões}

Conclui-se que o nutracêutico não influenciou nos níveis séricos de testosterona, cortisol e ganho de peso. Os parâmetros sanguíneos e do sêmen não sofreram alterações devido ao nutracêutico.

\section{Agradecimentos}

A Fazenda Aurora pelo apoio e financiamento desta pesquisa.

\section{Referências}

ALVES, P. A. P. M.; CAMPOS, O. F.; ALMEIDA, M. I. V.; LIZIEIRE, R. S.; MODESTA, R. C. D.; ALMEIDA, F. Q.; NASCIMENTO, C. G. H. Uso de probiótico composto por Lactobacillus acidophilus, Streptococcus faecium e Sacharomyces cerevisae na dieta de vitelos bovinos: efeitos sobre o desempenho e a qualidade da carne. Brazilian Journal Veterinary Research and Animal Science, São Paulo, v. 37, n. 5, p.21-27, 2000.
ARENAS, S. E.; REIS, L. S. L. S.; FRAZATTIGALLINA, N. M.; GIUFFRIDA, R.; PARDO, P. E. Efeito do probiótico Proenzime ${ }^{\circledR}$ no ganho de peso em bovinos. Archivos de Zooctecnia, v. 56, n. 213, p. 75-78, 2007.

AUDET, I.; LAFOREST, J. P.; MARTINEAU, G. P.; MATTE, J. J. Effect of vitamin supplements on some aspects of performance, vitamin status, and semen quality in boars. Journal of Animal Science, Quebec, v. 82, n. 2, p. 626-633, 2004.

BANZATTO, D. A.; KRONKA, E. S. N. Experimentação agricola. 4. ed. Jaboticabal: FUNEP. 2006. 237 p.

BARBOSA, R. T.; FONSECA, V. O.; BARBOSA, P. F.; ALENCAR, N. M. Concentrações plasmáticas e suas relações com características reprodutivas em touros das raças Canchim e Nelore. Revista Brasileira de Reprodução Animal, Belo Horizonte, v.16, n. 1, p.1-11, 1992.

BLOCKEY, M. A .B.; GALLOWAY, D. B. Hormonal control of serving capacity in bulls. Theriogenology, New York, v. 9, n. 2, p. 143-151, 1978.

CHACUR, M G. M.; MACHADO NETO, N. B. Influência da estação do ano sobre as proteínas do plasma seminal de touros Limousin. Veterinária Notícias, Uberlândia, v. 13, n. 1, p. 47-53, 2007.

CHACUR, M. G. M.; ARAÚJO, M. C.; KRONKA, S. N. Características seminais, corpóreas e anatômicas do aparelho reprodutor de reprodutores da raça Canchim aos 14 e 48 meses de idade. Arquivos de Ciências Veterinárias e Zoologia da UNIPAR, Umuarama, v. 9, n. 1, p. 21-27, 2006a.

CHACUR, M. G. M.; MACHADO NETO, N. B.; CRISTANCHO, D. R. Winter-spring and summer influence upon seminal plasma proteins in bulls. Animal Reproduction, Belo Horizonte, v. 3, n. 2, p. 251, 2006 b. 
CHACUR, M. G. M.; MACHADO NETO, N. B.; RABESQUINE, M. M. Season influence upon seminal plasma proteins in bulls. In: INTERNATIONAL CONGRESS ON ANIMAL REPRODUCTION, 15., 2004, Porto Seguro. Abstracts... Porto Seguro: Brazilian College of Animal Reproduction, 2004. p. 236. v. 1.

CHACUR, M. G. M.; RABESQUINE, M. M.; MACHADO NETO, N. B. Seleção da fertilidade em touros e proteínas do plasma seminal: correlação com o quadro espermático. Revista Brasileira de Reprodução Animal, Belo Horizonte, v. 27, n. 3, p. 185-186, 2003.

CHACUR, M. G. M.; SANCHEZ-MARTINEZ, A. I.; MACHADO NETO, N. B. Perfil em SDS-PAGE das proteínas do plasma seminal e sua relação com a qualidade do sêmen de touros da raça Nelore (Bos taurus indicus). Veterinária Notícias, Uberlândia, v. 12, n. 1, p. 87-93, 2006c.

CHACUR, M. G. M. Estresse térmico em touros bufalinos Bubalus bubalis, avaliações das características fisiológicas da reprodução. 2000. Tese (Doutorado em Medicina Veterinária). Departamento de Reprodução Animal e Radiologia Veterinária - Universidade Estadual Paulista, Botucatu. 2000.

CHACUR, M. G. M.; OBA, E. Heat stress in buffalo bulls Bubalus bubalis, evaluations of reproduction phisiologycal characteristics. Veterinária Notícias, Uberlândia, v. 11, n. 1, p. 111-112, 2005.

CHASE, C. C.; LARSEN, R. E.; HAMMOND, A. C.; RANDEL, R. D. Effect of dietary energy on growth and reproductive characteristics of Angus and Senepol bulls during summer in Florida. Theriogenology, Brooksville, v. 40, n. 1, p. 43-61, 1993.

\section{COLÉGIO BRASILEIRO DE REPRODUÇÃO} ANIMAL - CBRA. Manual para exame andrológico e avaliação de sêmen animal. 2. ed. Belo Horizonte: CBRA, 1998. 49 p.

DIAS JÚNIOR, R. F.; BRACARENSE, A. P. F. R. I.; MARÇAL, W. S.; ROCHA, M. A.; DIAS, R. C. F. Valores de referência e influência da idade no eritrograma de fêmeas bovinas da raça Aquitânica. Arquivo Brasileiro de Medicina Veterinária e Zootecnia, Belo Horizonte, v. 58, n. 3, p.311-315, 2006.

EVANS, A. C. O. Changes in circulating hormone concentrations, testes histology and testesultrasonography during sexual maturation in beef bulls. Theriogenology, Saskatoon, v. 46, n. 2, p. 345-357, 1996.

FELDMAN, B. E.; ZINKL, J. G.; JAIN, N. C. Schalm's veterinary hematology. 5 ed. Baltimore: Lippincott Williams \& Wilkins. 2000. 1244 p.
GABALDI, S. H. Alterações espermáticas e dos niveis plasmáticos de testosterona e cortisol em touros da raça Nelore, submetidos a insulação escrotal. 2000. Dissertação (Mestrado em Medicina Veterinária). Departamento de Reprodução Animal e Radiologia Veterinária - Universidade Estadual Paulista, Botucatu, 2000.

HAFEZ, E. S. E. Reprodução animal. 7. ed. São Paulo: Manole, 2004. $530 \mathrm{p}$.

HURLEY, W. C.; DOANE, R. M. Recent developments in the roles of vitamins and minerals in Reproduction. Journal of Dairy Science, Urbana, v. 72, n. 3, p. 784-804, 1989.

JORGE, C. F. J. F.; ROSA, G. O.; SILVA, I. S. Efeito de um aditivo alimentar contendo probiótico e enzimas digestivas no ganho de peso de bovinos Nelore em regime de pasto. In: ENCONTRO DE PESQUISA E INICIAÇÃO CIENTÍFICA DO ESTADO MATO GROSSO E DA REGIÃO DO PANTANAL, 4., 2006, Cuiabá. Anais... Cuiabá: EPICMT, 2006. p. 5.

KUMAR, N.; VERMA, R. P.; SINGH, L. P.; VARSCHNEY, V. P.; DASS, R. S. Effect of different levels and sources of zinc supplementation on quantitative and qualitative semen attributes and serum testosterone level in crossbred cattle (Bos indicus x Bos taurus) bulls. Reproduction Nutrition Development. v. 46, n. 6, p. 663675, 2006.

LEZIER, D. H. Avaliação da biometria testicular, concentração plasmática de hormônios e minerais em bovinos Nelore variedade mocha dos 12 aos 24 meses de idade. 2004. Tese (Doutorado em Medicina Veterinária). Departamento de Reprodução Animal e Radiologia Veterinária - Universidade Estadual Paulista, Botucatu. 2004.

LOWMAN, B. G.; SCOTT, N. A.; SOMERVALLE, L. H. Condition scoring. Edinburg: The East of Scotland College of Agriculture, 1976. $13 \mathrm{p}$.

LUCCI, C. S.; MOXON, A. L.; ZANETTI, M. A.; FUKUSHIMA, R. S.; SCHALCH, I.; PETTINATI, R. L. Selênio em bovinos leiteiros do estado de São Paulo: I. Níveis de selênio em soros sanguíneos. Revista da Faculdade de Medicina Veterinária e Zootecnia da Universidade de São Paulo, São Paulo, v. 21, n. 2, p. 65-70, 1984.

MARTINEZ L. M.; VERNEQUE, R. S.; TEODORO, R. L.; PAULA, L. R. O.; CRUZ, M.; CAMPOS, J. P.; RODRIGUES, L. H.; OLIVEIRA, J.; VIEIRA, F.; BRUSCHI, J. H.; DURÃES, M. C. Correlações entre características da qualidade do sêmen e circunferência escrotal de reprodutores da raça Gir. Revista Brasileira de Zootecnia, Viçosa, v. 29, n. 1, p. 1-15, 2000. 
McDOWELL, L. R.; EDWARDS, W.; GREENHALGH, A. Minerals in animal and human nutrition. San Diego: Academic Press. 1992. 524 p.

NATIONAL ACADEMY OF SCIENCE. Vitamin A. In: Dietary Reference Intakes for Vitamin A, Vitamin $\mathrm{K}$, Arsenic, Boron, Chromiun, Copper, Iodine, Iron, Manganese, Molybdenum, Nickel, Silicon Valadium and Zinc. cap. 4, p. 82-161, 2002. Disponível em: <www.nap. edu/openbook/0309072794/110.html.> Acesso em: $10 \mathrm{de}$ out. 2008.

NATIONAL RESEARCH COUNCIL - NRC. Nutrient requerements of dairy cattle. 7. ed. Washington: National Academy Press. 2001. 381 p.

OBA, E. Estudo das características quantitativas e qualitativas do soro sanguíeo e do sêmen de bovinos Nelore em diferentes idades. 1985. Tese (Doutorado em Medicina Veterinária). Departamento de Reprodução Animal e Radiologia Veterinária-Universidade Estadual Paulista, Botucatu, 1985.

PASCHOAL, J. J.; ZANETTI, M. A.; CUNHA, J. A. Efeito da suplementação de selênio e vitamina E sobre a incidência de mastite clínica em vacas da raça Holandesa. Arquivo Brasileiro de Medicina Veterinária e Zootecnia. Belo Horizonte, v. 55, n. 3, p. 349-255, 2003.

POST, T. B.; CHRISTENSEN, H. R.; SEIFERT, G. W. Reproductive performance and produtive in beef bulls selected for different leves of testosterone response to GnRH. Theriogenology, New York, v. 27, n. 2, p. $317-$ 328, 1987.

RABESQUINE, M. M.; CHACUR, M. G. M.; GARCIA, J. P. Morfometria testicular, aspectos seminais e influência do peso corpóreo sobre a morfologia espermática na raça Limousin. Revista Brasileira de Reprodução Animal, Belo Horizonte, v. 27, n. 2, p. 185-186, 2003.

RASTEIRO, V. S.; BREMER-NETO, H.; ARENAS, S. E. Adição de probiótico na mistura mineral eleva o ganho de peso de bovinos no período da seca. In: REUNIÃO ANUAL DA SOCIEDADE BRASILEIRA DE ZOOTECNIA, 43., 2006, João Pessoa. Anais... João Pessoa: SBZ, 2006. p. 83-87.

RODE, L. M.; COULTER, H. G.; KASTELIC, J. P.; BAILEY, D. R. C. Seminal quality and sperm production in beff bulls with chronic dietary vitamin A deficiency and subsequent re-alimentation. Theriogenology, Lethbridge, v. 43, n .7, p. 1269-1277, 1995.

SANCHES, A. C. Concentração plasmática de testosterona e suas relações com características reprodutivas e morfométricas de touros jovens das raças
Nelore e Santa Gertrudis. Dissertação (Mestrado em Ciências Biológicas). Departamento de Morfologia Universidade de São Paulo, Riberão Preto, 1999.

SANCHES, A. C.; LOBO, R. B.; BEZERRA, F. L. A.; MACARI, M. Variação da secreção de testosterona no desenvolvimento corporal e idade à puberdade em touros Bos indicus. In: REUNIÃO DA SOCIEDADE BRASILEIRA DE ZOOTECNIA, 35., 1998, Botucatu. Anais.... Botucatu: SBZ, 1998. p. 13-15.

SANCHEZ, A. I.; CHACUR, M. G. M.; COUTINHO, N. V. Semen physical and morphological characteristics and corporal mass index of Nelore (Bos taurus indicus). In: INTERNATIONAL CONGRESS ON ANIMAL REPRODUCTION, 15, 2004, Porto Seguro. Abstracts... Porto Seguro: Brazilian College of Animal Reproduction, 2004. v. 1, p. 196.

SANTOS, M. D. Comportamento sexual, qualidade seminal e eficiência reprodutiva de touros da raça Nelore em regime de monta natural. 1999. Tese (Doutorado em Zootecnia). Departamento de Produção Animal Universidade Federal de Viçosa, 1999.

SARREIRO, L. C. Herdabilidade e correlação genética entre perímetro escrotal, libido e características seminais de touros Nelore. Arquivo Brasileiro de Medicina Veterinária e Zootecnia. Belo Horizonte. v. 54, n. 2, p. 602-608, 2002.

SETCHELL, B. P. The effects of heat on the testes of mammals. Animal Reproduction, Belo Horizonte, v. 3, n. 2, p. 81-91, 2006.

SILVA, A. Capacidade reprodutiva do touro de corte: funções, anormalidade e fatores que a influenciam. Campo Grande: Empresa Brasileira de Pesquisa Agropecuária, 1993. Relatório.

SILVA, A. E. D. F.; UNANIAN, M. M.; CORDEIRO, C. M. T.; FREITAS, A. R. Relação da circunferência escrotal e parâmetros da qualidade do sêmen em touros da raça Nelore, PO. Revista Brasileira de Zootecnia, Viçosa, v. 31, n. 3, p. 1157-1165, 2002.

WANG, R.; ZHU, X.; GUO, F.; ZHANG, W.; JIA, Z. Influence of different dietary levels of zinc performance, vitamin B12 and blood parameters in lambs. International Journal of Vitamine and Nutrition Research, Beijing, v. 76, n. 6, p.353, 2006.

WELLIK, D.; DELUCA, F. H. Metabolites of all-transretinol in day 10 conceptus of vitamin A - deficient rats. Archives of Biochemistry and Biophysical, London, v. 33, n.3, p. 355-362, 1996. 
